\title{
Oxidative stress, immunological and cellular hypoxia biomarkers in hepatitis C treatment-naïve and cirrhotic patients
}

\author{
Abdulrahman Hamdan Almaeen ${ }^{1}$, Abdulrahman Abdulwahab Alduraywish², \\ Maysa Ahmed Mobasher ${ }^{1}$, Omar I. M. Almadhi ${ }^{3}$, Hanan M. Nafeh4, Tarek Hassan El-Metwally ${ }^{1,5}$
}

\author{
'Department of Pathology, Jouf University College of Medicine, Sakaka, Saudi Arabia \\ 2Department of Internal Medicine, Jouf University College of Medicine, Sakaka, \\ Saudi Arabia \\ ${ }^{3}$ College of Medicine, Jouf University College of Medicine, Sakaka, Saudi Arabia \\ ${ }^{4}$ Department of Tropical Medicine and Gastroenterology, Assiut University, \\ Faculty of Medicine, Assiut, Egypt \\ ${ }^{5}$ Department of Medical Biochemistry, Assiut University, Faculty of Medicine, Assiut, \\ Egypt
}

Submitted: 25 August 2019; Accepted: 13 November 2019;

Online publication: 3 January 2020

Arch Med Sci 2021; 17 (2): 368-375

DOI: https://doi.org/10.5114/aoms.2019.91451

Copyright @ 2019 Termedia \& Banach

\begin{abstract}
Introduction: Hepatitis C virus (HCV) is the main cause of chronic liver disease, with calamitous complications. Its highest rate is recorded in Egypt. This study investigated whether oxidative stress, immunological chaos and cellular hypoxia are implicated in the pathophysiology of the disease.

Material and methods: This cross-sectional study aimed to evaluate the changes in blood oxidative stress, cellular hypoxia/angiogenesis and cellular immunological biomarkers in hospital-diagnosed treatment-naïve HCV-infected Upper Egyptian chronic liver disease patients vs. healthy controls $(n=40)$. The consecutively included patients comprised 120 with normal serum enzymes (HCV-NE) and 130 with high serum enzymes (HCV-HE), along with 120 cirrhotic patients.

Results: Oxidative stress biomarkers - malondialdehyde (MDA), total peroxides and oxidative stress index (OSI) - were significantly lower in controls vs. each of the patient groups. Cirrhotic patients presented the highest levels. However, total antioxidants (TAO) showed non-significant differences among the four groups. The cellular hypoxia/angiogenesis biomarkers - lactate, vascular endothelial cell growth factor (VEGF) and its soluble receptor 1 (sVEGFR1) - vs. controls were massively increased in patient groups. VEGF was lowest while SVEGFR1 was highest among cirrhotic patients. Immunological biomarkers, - granulocyte/monocyte-colony stimulating factor (GM-CSF) and total immunoglobulin $\mathrm{G}(\operatorname{IgG})$ - were massively increased in patient groups vs. controls. GM-CSF was lowest in HCV-HE and IgG was highest in cirrhotic patients. SVEGFR1 correlated with the progression towards cirrhosis.

Conclusions: Oxidative stress is implicated in the progress of HCV infection with marked induction of cellular hypoxia and dysfunctional angiogenesis, and a futile immunological reaction. SVEGFR1 level correlated with progression towards HCV-induced liver fibrosis.
\end{abstract}

Key words: oxidative stress, liver cirrhosis, liver enzymes, hypoxia, hepatitis $C$ virus, chronic liver disease, immunological changes.
Corresponding author: Prof. Tarek Hassan ElMetwally Division of Biochemistry Department of Pathology College of Medicine Jouf University Sakaka, Saudi Arabia Phone: +96 6541860565 E-mail: thelmetwally@ hotmail.com 


\section{Introduction}

Without effective vaccination, progressive hepatitis $\mathrm{C}$ virus (HCV) represents a major global health problem. It predisposes to the development of hepatic and extrahepatic complications and mortality [1, 2]. Worldwide, particularly among the population aged 15 years or over, Egypt has the highest prevalence. Therein, the prevailing genotype is 4, which is the most aggressive and highly associated with severe fibrosis and transformation [3]. Despite the accumulation of a large body of data, HCV pathogenesis requires further scrutiny.

One pathogenic mechanism for HCV is oxidative stress resultant from increased generation of reactive oxygen species in infected hepatocytes, where redox homeostatic balance is a major challenge to prevent cell death and DNA mutation [4-8]. Mechanistically, HCV activates prooxidant enzymes and hepatic stellate cells, weakens antioxidant defenses, and induces organelle damage, secretion of pro-inflammatory cytokines and metals unbalance - secondary to mitochondrial failure, which is a focal point in that scenario $[9,10]$. The early induced oxidative stress is responsible for HCV liver disease progression towards both steatosis and fibrosis [9].

Liver injury disturbs hepatic architecture and hinders blood flow that is further deteriorated by the locally induced vaso-/angioactive compounds. The resultant multiple areas of hypoxia increase lactate accumulation and reduce its clearance. In such a scenario, hypoxia seems to be a systemic as well as a local problem, as reflected by the induced systemic angiogenesis [11-16]. Hypoxia-induced vascular endothelial growth factors (VEGF) and their counteracting soluble receptors (sVEGFRs) are major pathophysiological players. Their inducibility is orchestrated by the transcription regulator, the hypoxia-inducible factor-1 $\alpha$ (HIF-1 $\alpha$ ) [17-20]. HCV infections promote oxidative stress, while downregulating VEGF mRNA expression very early after infection that rebounds in the long term [9]. The marked variability in VEGF and sVEGFR concentrations in HCV-induced hepatic disease makes them weak indicators for the progression of chronic liver disease in cirrhotic patients. However, they may reflect the increases in portal hypertension and/or the decreases in hepatic regenerative activity [18-21]. Moreover, the interaction between the virus and the immune system response is a critical pathogenic mechanism. In chronic liver disease, abnormal immunological responses include autoantibodies, hypergammaglobulinemia, cytokine profile changes and oxidative stress, especially in decompensated hepatic cirrhosis [21].

Diagnostic blood markers and non-invasive imaging methods for determining liver dam- age and its pathogenesis are being validated. The target of this study was to investigate the changes in oxidative stress, cellular hypoxia/ angiogenesis and immunological biomarkers in blood of Upper Egyptian patients with treatment-naïve HCV infection (with normal vs. high enzyme levels) and liver cirrhosis compared to healthy volunteers. The correlations between these biomarkers and the liver state were analyzed as potential pathogenic effectors and/or diagnostic biomarkers.

\section{Material and methods}

\section{Setting}

This cross-sectional study voluntarily enrolled consented patients and their age- and socio-economically matching healthy companions attending the Hepatology Institute, Assiut University Hospitals, Assiut, Egypt, in the period from January 1, 2016 to December 1, 2018. The local Bioethics Committee of the Faculty of Medicine, Assiut University approved this study. De-identified demographic data and disease history were collected from patients' records. Patients' exclusion criteria included immobilization for any reason, renal failure, autoimmune diseases, acute infections, congenital and hemolytic anemia and systemic inflammatory diseases.

370 treatment-naïve patients with HCV-related chronic liver disease were consecutively included in our study. They encompassed three groups: 120 with normal serum liver enzymes (HCV-NE; mean \pm SDM, ALT $=32.75 \pm 10.91$; AST $=35.75$ $\pm 7.01 ; \mathrm{APL}=70.29 \pm 15.19 \mathrm{U} / \mathrm{l}), 130$ with high serum enzymes (HCV-HE; ALT = $120.5 \pm 83.97$; $\mathrm{AST}=85.1 \pm 45.87 ; \mathrm{APL}=120.5 \pm 44.85 \mathrm{U} / \mathrm{l} ; p<$ 0.001 ), and 120 cirrhotic patients. Hospital-based diagnosis - through thorough clinical examination, abdominal ultrasonography, liver function tests and liver biopsy - was done to characterize the chronic liver disease. HCV infection was diagnosed based on specific positive anti-HCV serological testing and RT-PCR assessed positive HCV RNA. Forty healthy controls were enrolled for comparison. The male-female ratio in all groups was $1: 1$.

\section{Samples and investigations}

Serum was recovered from fasting peripheral blood samples collected in plain tubes after clotting and centrifugation. Serum was aliquoted and stored at $-70^{\circ} \mathrm{C}$. Plasma recovered from another fasting heparin/sodium fluoride peripheral blood sample was immediately deproteinized with two volumes of perchloric acid for enzymatic measurement of lactate (Cat. No. 16300, Greiner Diagnostic $\mathrm{GmbH}$, Bahlingen, Germany). Serum to- 
tal IgG was measured using a SPAPLUS kit (Code NK004.S, The Binding Site Group Ltd, Birmingham, UK). Using a specific commercially available ELISA kit, serum granulocyte-macrophage colony-stimulating factor (GM-CSF) was measured (Cat. No. KAC0901, BioSource Europe S. A., Nivelles, Belgium). Quantikine VEGF immunoassay kits were used to measure serum VEGF and SVEGFR1 (Cat. \# RRV00 and \# DVR100B, R\&D Systems, Inc., MN, USA). Serum total peroxides were measured colorimetrically as $\mathrm{H}_{2} \mathrm{O}_{2}$ equivalents by xylenol orange reagent [22]. We used 2,2'-azinobis-3-ethylbenzothiazoline-6-sulfonate (ABTS) for the colorimetric measurement of serum total antioxidants (TAOs) as Trolox equivalents [23]. Serum oxidative stress index (OSI) was calculated as a percent ratio of TAO content in $\mathrm{mM}$ to total peroxides in $\mathrm{mM}$ of each sample [22]. Serum malondialdehyde (MDA), a lipid peroxidation and oxidative stress biomarker, was assayed colorimetrically using 2-thiobarbituric acid as 1,1,3,3-tetraethoxypropane precursor equivalents [24].

\section{Statistical analysis}

Data are presented as frequency $(n)$ and mean \pm SDM. Prism 6.0 GraphPad (GraphPad Software, Inc., La Jolla, CA, USA) was used for the statistical analysis. One-way ANOVA with the Newman-Keuls multiple comparison test was applied. Correlation among parameters within groups was analyzed using Spearman's nonparametric correlation analysis. The significance limit was set at a $p$-value of $\leq 0.05$ at a $95 \%$ confidence interval.

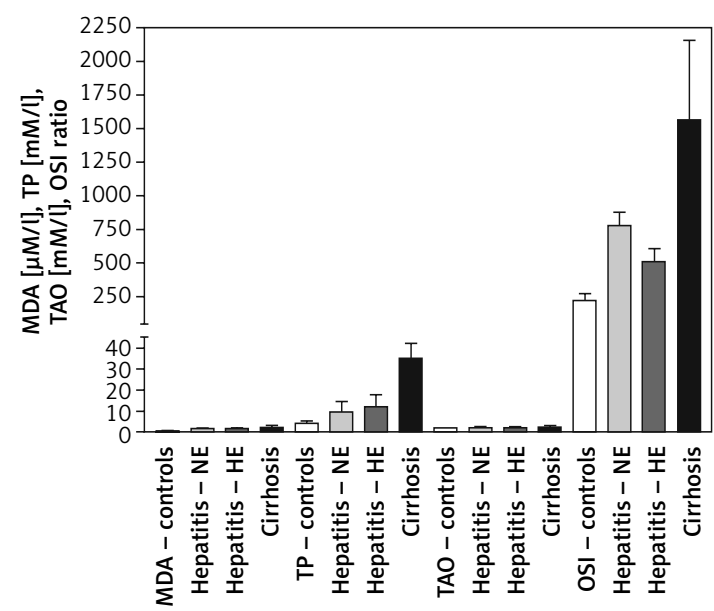

Figure 1. Changes in serum oxidative stress biomarkers of Egyptian patients with HCV infection (with normal vs. high enzyme levels) and liver cirrhosis vs. healthy controls. Data shown are mean \pm SDM

MDA - malondialdehyde, TP - total peroxides, TAO total antioxidants, OSI - oxidative stress index, NE - HCV infection with normal serum enzymes, HE - HCV infection with high serum enzymes.

\section{Results}

\section{Changes in oxidative stress biomarkers}

MDA levels were $0.56 \pm 0.12,1.6 \pm 0.21,1.62$ \pm 0.37 , and $1.934 \pm 0.622 \mu \mathrm{M} / \mathrm{l}$ for the healthy controls, HCV-NE, HCV-HE, and cirrhotic patients, respectively. This revealed markedly higher MDA levels in the three patient groups compared to the healthy controls $(p<0.001)$. There were no significant differences among the patient groups. Total peroxide levels for controls, HCV-NE, HCV-HE and cirrhotic patients were $4.0 \pm 1.0,9.41 \pm 5.08$, $12.11 \pm 5.64$, and $35.1 \pm 7.303 \mathrm{mM} /$, respectively. This showed significantly higher total peroxides in all patient groups vs. healthy controls $(p<0.001)$. A small, significant increase was observed comparing HCV-NE and HCV-HE patients ( $p<0.05$ ). Cirrhotic patients revealed markedly higher levels of total peroxides vs. the other patient groups ( $p<0.001)$. TAOs levels of $1.89 \pm 0.1,2.03 \pm 0.54$, $1.89 \pm 0.56$, and $2.249 \pm 0.611 \mathrm{mM} / \mathrm{I}$ for the healthy controls, HCV-NE, HCV-HE, and cirrhotic patients (respectively) were non-significantly different. OSI was $220 \pm 52.6,778.4 \pm 100.8$, $508.6 \pm 98.16$, and $1560.7 \pm 595.3$ for controls, HCV-NE, HCV-HE, and cirrhotic patients, respectively. Because of the marked increase in total peroxides, this OSI was significantly lower in controls vs. each of HCV-NE, $\mathrm{HCV}-\mathrm{HE}$, and cirrhotic patients $(p<0.001,0.05$, and 0.001 , respectively). HCV-NE and HCV-HE groups were significantly different $(p<0.05)$, and each of them was significantly lower than cirrhotic patients $(p<0.001)$ (Figure 1$)$.

\section{Changes in blood hypoxia and angiogenesis biomarkers}

Plasma lactate levels were $1.3 \pm 0.447,2.98$ $\pm 0.74,3.39 \pm 1.71$, and $3.959 \pm 1.127 \mathrm{mM} / \mathrm{l}$ for controls, HCV-NE, HCV-HE, and cirrhotic patients, respectively. Lactate was significantly lower in controls compared to all patient groups ( $p<0.001)$. Patient groups were non-significantly different. VEGF levels were $0.22 \pm 0.08,9.1 \pm 1.27$, $8.56 \pm 0.79$, and $7.705 \pm 0.785 \mathrm{ng} / \mathrm{ml}$ in controls, HCV-NE, HCV-HE and cirrhotic patients, respectively. This indicated the massively increased levels in all patient groups compared to controls ( $p<0.001)$. Compared to HCV-NE and HCV-HE groups, cirrhotic patients had lower VEGF levels ( $p<0.001$ and 0.05 , respectively). In controls, HCV-NE, HCV-HE and cirrhotic patients the serum sVEGFR1 levels were $114 \pm 22,439.1 \pm 15.35$, $469 \pm 23.9$, and $542 \pm 52 \mathrm{pg} / \mathrm{ml}$, respectively. This demonstrated that sVEGFR1 levels were more than tripled in all patient groups vs. controls $(p<0.001)$. Significantly higher levels of serum sVEGFR1 were noted in the cirrhotic patients than the other two patient groups $(p<0.001)$. HCV-HE 
patients exhibited higher serum SVEGFR1 levels compared to the HCV-NE group $(p<0.05)$ (Figure 2).

\section{Changes in serum immunological biomarkers}

Serum GM-CSF levels were $7.62 \pm 4.92,370$ $\pm 117,227 \pm 87$, and $388 \pm 152 \mathrm{mM} / \mathrm{l}$ for controls, HCV-NE, HCV-HE, and cirrhotic patients, respectively. This indicated that serum GM-CSF level was increased to more than 25-fold the control levels in the patient groups $(p<0.001)$. HCV-NE and cirrhotic patients had a higher GM-CSF level vs. HCV-HE patients $(p<0.01)$. Serum total IgG levels in controls, HCV-NE, HCV-HE and cirrhotic patients were $9.299 \pm 2.358,15.4 \pm 2.1,14.2 \pm 1.37$, and $18.58 \pm 3.596 \mathrm{~g} / \mathrm{l}$, respectively. Patient groups showed markedly higher levels of IgG than controls ( $p<0.001)$. Compared to both HCV groups, cirrhotic patients had significantly higher levels of serum total IgG $(p<0.001)$, and patients with HCV-HE showed significantly lower contents compared to patients with HCV-NE $(p<0.01)$ (Figure 3).

\section{Correlation analysis among parameters}

Correlations among all parameters were nonsignificant in healthy controls. In HCV-NE patients, total peroxides and MDA correlated positively $(r=0.643, p<0.05)$, and sVEGFR1 vs. IgG $(r=-0.689, p<0.05)$ and TAOs vs. MDA $(r=-0.664$, $p<0.05)$ showed significant negative correlations. In HCV-HE patients, there were significant positive correlations between lactate and each of VEGF $(r=0.622, p<0.05), \mathrm{GM}-\mathrm{CSF}(r=0.718, p<0.01)$

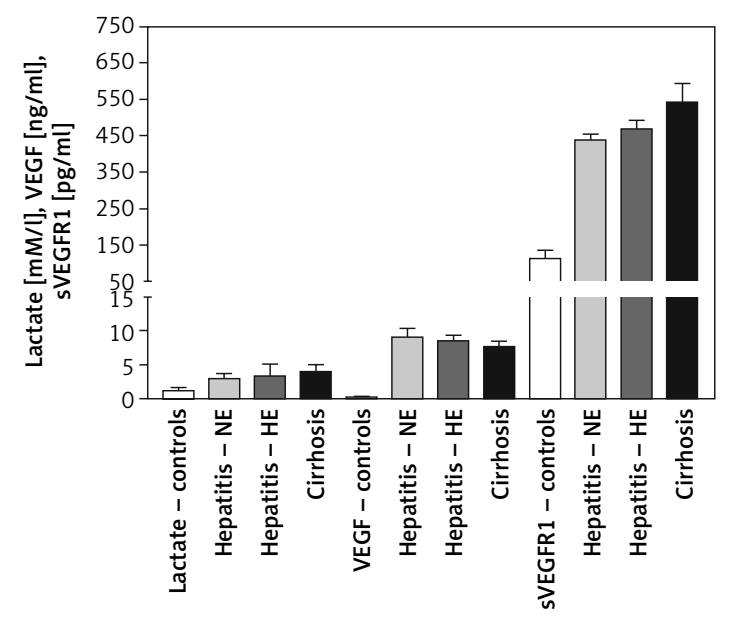

Figure 2. Changes in blood cellular hypoxia/angiogenesis biomarkers in Egyptian patients with HCV infection (with normal vs. high enzyme levels) and liver cirrhosis vs. healthy controls. Data shown are mean \pm SDM

VEGF - vascular endothelial cell growth factor, SVEGFR1 soluble VEGF receptor 1 . and IgG $(r=0.689, p<0.05)$, between VEGF and GM-CSF $(r=0.660, p<0.05)$, between sVEGFR1 and each of GM-CSF $(r=0.602, p<0.05)$ and IgG $(r=0.522, p<0.05)$, and between TAOs and IgG $(r=0.484, p<0.05)$. In cirrhotic patients, there were significant positive correlations between VEGF and each of sVEGFR1 $(r=0.650, p<0.05)$, total peroxides $(r=0.619, p<0.05)$ and lactate $(r=0.700, p<0.05)$; between TAOs and each of total peroxides $(r=0.887, p<0.01)$ and GM-CSF $(r=0.676, p<0.05)$, and between lactate and each of sVEGFR1 $(r=0.733, p<0.05)$ and MDA $(r=0.778, p<0.01)$.

\section{Discussion}

The blood-borne hepatitis $C$ virus infection is the most common cause of chronic viral hepatitis in the world, including Egypt [25]. In the absence of an in vitro system that recapitulates the in vivo stages of HCV infection, the pathogenic mechanisms leading to the hepatocellular damage are not fully understood. However, it is established that not only does HCV infection induce oxidative stress, but also the replication of the HCV virus depends on such redox-sensitive cellular changes [26]. Biomarkers of oxidative stress are increased in liver tissue and body fluids of patients with chronic HCV infection. Induction of oxidative stress correlates with the incidence and/or severity of HCV-related morbidities, since chronic HCV infection relies on this strategy for immune escape and persistence [5-8]. In support, antioxidants were promising co-adjuvants in HCV therapy [27]. In the same direction, our results

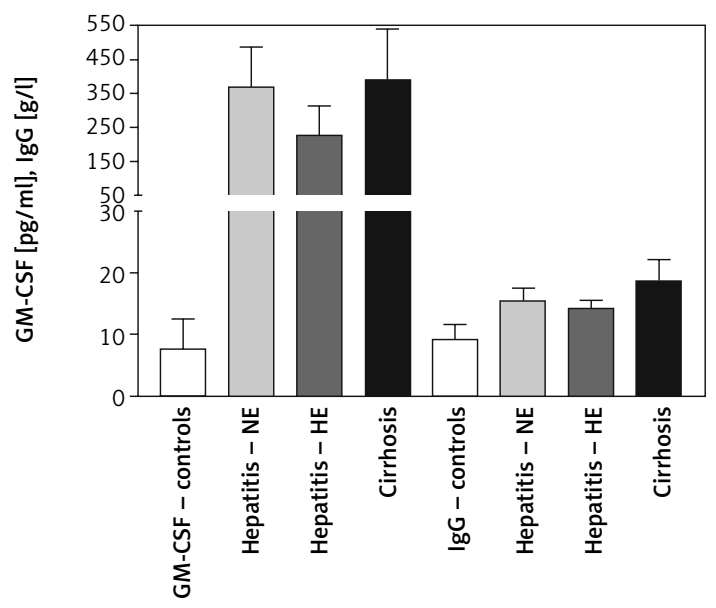

Figure 3. Changes in serum immunological biomarkers in Egyptian patients with HCV infection (with normal vs. high enzyme levels) and liver cirrhosis vs. healthy controls. Data shown are mean \pm SDM

GM-CSF - granulocyte/monocyte-colony stimulating factor, IgG - total immunoglobulin $G$. 
revealed marked oxidative stress in HCV patients caused mainly by increases in oxidants rather than decreases in antioxidants. MDA level was significantly higher in patient groups compared to controls, which in part reflects the continuing cellular damage in response to HCV. Reportedly, MDA level and its protein adducts are elevated in liver and blood samples of chronic HCV patients and correlated positively with the stage of hepatic fibrosis and negatively with the response to treatment [28]. Moreover, ischemia-reperfusion, which simulates hypoxia and the HIF-1 $\alpha$-orchestrated response, increases tissue MDA and induces pronounced changes in hepatic architecture due to steatosis and cell destruction [15]. Similarly, our patients also showed a significant increase in OSI due to the increased total peroxides, and HCV-NE patients revealed a significant positive correlation between MDA and total peroxides. This was reasoned to be due to inflammation and mitochondrial dysfunction [29]. Two possible explanations for the non-significant changes in TAOs among all groups are: 1) induction of antioxidant mechanisms because of the disease to counteract the generated oxidative stressors, and 2) the increases in the highly antioxidant bilirubin in patients. TAOs and MDA showed a significant negative correlation in HCV-NE patients similar to previous studies $[6,9,11,28]$. In the cirrhotic patients, there was a positive significant correlation between TAOs and total peroxides. Therefore, the redox homeostatic imbalance caused the pro-oxidant status and oxidative stress in our patients despite the increases in antioxidants. Reportedly, reactivation of $\mathrm{HCV}$ viral replication could favor the prooxidant state that exacerbates cell damage and death [26].

Lactate is produced in the peripheral tissues under normal physiological conditions, and is metabolized mainly in the liver and kidneys. HCV infection induces transcriptional downregulations that affects mainly mitochondrial respiratory chain complex core subunit genes. This induces metabolic reprogramming and aerobic glycolysis with lactate accumulation and enhanced generation of reactive oxygen species $[11,16]$. HCV viral-protein expression and infection severely impair mitochondrial oxidative phosphorylation leading to major reliance on nonoxidative glucose metabolism. This occurs through induction of HIF-1 $\alpha$ and its dependent VEGF and glycolytic enzymes, in vitro and in vivo [30-32]. Interestingly, such hypoxic conditions and associated gene expression changes enhance HCV replication. Vice versa, inhibition of HIF- $1 \alpha$ reduced HCV replication; therefore, they are pathogenic in the disease process [12-14]. Consequently, prolongation of lactate elimination in cirrhotic patients compared to normal individuals is rationally expected [33]. Moreover, such sim- ple lactemia turns into aggressive lactic acidosis upon HCV antiviral treatment [34]. In agreement, our observation of a significant increase in blood lactate and a positive correlation between lactate and MDA in cirrhotic patients indicate that they both reflect hepatocellular damage [33].

Experimental models of portal hypertension and liver cirrhosis inferred a direct role for angiogenesis. Among a long list of angiogenic modulators, VEGF is the most potent angiogenic and vasculogenic mediator. The cellular signaling machinery is exploited by human viruses to facilitate their own pathogenesis through upregulating the expression of VEGF [35]. VEGF is up-regulated in experimental and clinical cirrhotic livers. HCV infection accelerates angiogenesis by stabilizing HIF-1 $\alpha$ under normoxic growth conditions that correlates with increased VEGF secretion and membrane receptor expression [36-38]. Our study demonstrated that, compared to healthy controls, our HCV patients show significantly higher serum levels of VEGF but serum aminotransferases did not correlate with VEGF in patients. Consequently, it was established that the enhanced regenerative capacity of hepatocytes rather than the hepatocyte damage per se is the cause of the increased serum VEGF $[30,31]$. The clinical utility of sVEGFR1 as a pathogenic, diagnostic and/or prognostic marker in different medical conditions, including liver cirrhosis, either separately or accompanied with VEGF, was previously reported [39]. Our results are in concordance with such a notion; we observed elevated VEGF and over 3-fold increase in its antagonistic sVEGFR1 in all patient groups vs. controls. These changes are associated with the degree of hepatic insufficiency [39]. We found significant positive correlations in the cirrhotic patients between VEGF and each of total peroxides and lactate. This indicates that the triad of oxidative stress, hepatocellular dysfunction and angiogenesis/hypoxia are intertwiningly implicated. Similarly, we found significant correlations between lactate and VEGF in HCV hepatitis with high serum enzymes. Moreover, we observed significant positive correlations in the cirrhotic group between VEGF and SVEGFR1, and between lactate and SVEGFR1. These results confirmed the earlier findings of a dramatic elevation in VEGFA serum levels in HCV patients. Similar findings connected angiogenesis, as overexpressed VEGFA transcripts, with the progression of chronic liver diseases into fibrosis [40-42]. In cirrhotic patients as compared to healthy controls, over 3-fold increases in VEGF and SVEGFR1 levels were reported. Patients with the advanced stages of liver cirrhosis recorded the highest concentrations [39]. Serum sVEGFR1 increased to more than 3-fold the healthy control level in HCV-infected patients with hepatocellular carcinoma and correlated with the liver enzyme 
level and tumor stage in Egyptian patients [17]. Egyptian HCV-infected patients showed dramatically elevated serum levels of VEGF compared to healthy controls [43]. Compared to healthy controls, HCV patients had significantly higher VEGFA in peripheral blood mononuclear cells and serum [19]. Such levels confirmed the other international studies and correlated with the fibrosis grade in HCV-induced liver fibrosis [19, 20]. Egyptian patients with HCV-related liver cirrhosis had about 3-fold increases in serum VEGF that positively correlated with liver enzyme levels [44]. Chronic HCV with cirrhosis revealed significantly lower VEGF but higher SVEGFR1 levels compared to controls [18]. There was no significant difference comparing serum VEGF levels in patients with chronic HCV infection and those with HCV-related liver cirrhosis [45]. In patients with chronic hepatitis and liver cirrhosis, serum sVEGFR-2 correlated with the disease severity [46]. Egyptians with genotype-4 chronic HCV infection showed a moderate but significant increase in serum VEGF [19]. A Turkish study reported more than 3-fold increase in serum VEGF in HCV infection and liver cirrhosis patients that inversely correlated with fibrosis and cirrhosis scores [21]. The discrepancies among these reports could be related to variations in the disease course (early vs. chronic), viral load and activity, virus genotype and genetic background of patients.

HCV-induced progressive liver disease deteriorates from chronic inflammation to fibrosis and cirrhosis. Such a persistent uncontrolled inflammatory state worsens the hepatic damage. Changes in the inflammatory chemokine and cytokine profiles parallel the progress of HCV infection and its response to treatment. Therefore, changes in chemokines and cytokines are being validated as biomarkers for disease progression, its therapeutic responses and drug targets [47]. Moreover, the natural course of infection and the outcome of antiviral treatment are determined by the nature of the cytokine response against HCV [48]. Because of that, coupling of the direct-acting anti-HCV drugs with anti-inflammatory/hepatoprotective agents yields a better therapeutic prognosis [47].

GM-CSF regulates inflammation, resistance to viral infections and stimulation of tissue repair [49-51]. It stimulates the production of granulocytes and monocyte-macrophages [52]. GM-CSF serum level was increased to more than 25 -fold the healthy control levels in our patients. These results are in line with a previously reported Egyptian study that showed significant elevation in GM-CSF among chronic HCV patients [53]. Moreover, the increases in GM-CSF were correlated with increased lactate accumulation [54]. Interestingly, our results showed significant correlations between GM-CSF and each of lac- tate, VEGF, and SVEGFR1 in HCV patients with high serum enzymes. Furthermore, our cirrhotic patients revealed a significant positive correlation between GM-CSF and TAOs. Reportedly, compared to subjects in whom therapy was unsuccessful, the pretreatment cytokine response (including GM-CSF) was higher in patients who achieved a sustained virological response. Levels of histological grading and serum enzymes also positively correlated with the expression of GM-CSF, among other cytokines [48]. While serum enzymes were associated with the histologic activity index, GM-CSF was associated with a change in fibrosis stage [55]. However, a Brazilian study showed lower serum GM-CSF levels in chronic HCV patients than healthy controls. This may reflect the prevailing genotype and natural course of the disease [56]. GM-CSF has pro-inflammatory (granulocyte and macrophage growth and differentiation) and anti-inflammatory/regulatory (dendritic cell differentiation and increased regulatory T-cell numbers) effects. However, these effects depend on the presence and dose of other relevant cytokines in the immune response milieu. GM-CSF enhances the anti-HCV immune response induced by the HCV nonstructural protein NS3 [57]. This implicates the changes in the immunological cytokine profile as the fourth dimension in the pathogenic vicious tetrad of highly hepatotoxic feed-forward cycle of inflammation and cell death - alongside oxidative stress, hepatocellular dysfunction and angiogenesis/hypoxia. Reportedly, the abnormal immunological responses, as a feature of chronic HCV-induced liver disease, include autoantibodies and hypergammaglobulinemia - especially in decompensated hepatic cirrhosis [58]. Hypergammaglobulinemia is pathogenically causative in the progression of liver disease and advanced forms of hepatic fibrosis [59, 60]. In support, total serum levels of IgG in our study showed significantly higher levels in all patient groups compared to controls. Along with the futile increased production, this could be due to reduction in the rate of clearance of immunoglobulins by the diseased liver [61]. Interestingly, in HCV$\mathrm{HE}$ hepatitis, there were significant positive correlations between IgG and each of lactate, TAOs, and SVEGFR1, indicating the intimate interaction of the four pathogenic mechanisms investigated in our study. In the milder HCV-NE hepatitis, the correlation analysis revealed negative significant correlations between sVEGFR1 and IgG.

In conclusion, we have demonstrated the intimate pathogenic interactions among the significantly induced oxidative stress, immunological, and cellular hypoxia/disordered angiogenesis in our HCV hepatitis and cirrhosis patients. Our data 
further highlighted the potential prognostic and druggable value of most of these biomarkers, particularly sVEGFR1 in HCV-induced liver fibrosis.

\section{Conflict of interest}

The authors declare no conflict of interest.

\section{References}

1. Lozano R, Naghavi M, Foreman K, et al. Global and regional mortality from 235 causes of death for 20 age groups in 1990 and 2010: a systematic analysis for the Global Burden of Disease Study 2010. Lancet 2012; 380: 2095-128.

2. Applegate TL, Fajardo E, Sacks JA. Hepatitis C virus diagnosis and the holy grail. Infect Dis Clin North Am 2018; 32: 425-45.

3. Kandeel A, Genedy M, El-Refai S, Funk AL, Fontanet A, Talaat $M$. The prevalence of hepatitis $C$ virus infection in Egypt 2015: implications for future policy on prevention and treatment. Liver Int 2017; 37: 45-53.

4. Ivanov AV, Bartosch B, Smirnova OA, Isaguliants MG, Kochetkov SN. HCV and Oxidative stress in the liver. Viruses 2013; 5: 439-69.

5. Rebbani K, Tsukiyama-Kohara K. HCV-induced oxidative stress: Battlefield-Winning strategy. Oxid Med Cell Longev 2016; 2016: 7425628.

6. Ríos-Ocampo WA, Navas MC, Faber KN, et al. The cellular stress response in hepatitis $C$ virus infection: a balancing act to promote viral persistence and host cell survival. Virus Res 2019; 263: 1-8.

7. Zhang Z, Rong L, Li YP. Flaviviridae viruses and oxidative stress: implications for viral pathogenesis. Oxid Med Cell Longev 2019; 2019: 1409582.

8. Vuppalanchi R, Juluri R, Bell LN, et al. Oxidative stress in chronic liver disease: relationship between peripheral and hepatic measurements. Am J Med Sci 2011; 342: 314-7.

9. Saeed U, Piracha ZZ, Manzoor S. Hepatitis C virus induces oxidative stress and DNA damage by regulating DNAPKCS, ATM, ATR and PARP mediated signaling and guards cell from cancerous condition by upregulating RB, P53 and downregulating VEGF. Acta Virol 2017; 61: 316-23.

10. Arciello M, Gori M, Balsano C. Mitochondrial dysfunctions and altered metals homeostasis: new weapons to counteract HCV-related oxidative stress. Oxid Med Cell Longev 2013; 2013: 971024.

11. Chapplain JM, Tattevin P, Guyader D, et al. Mitochondrial abnormalities in patients with HIV-HCV co-infection as compared to patients with HCV mono-infection. HIV Clin Trials 2011; 12: 54-60.

12. Abe M, Koga H, Yoshida T, et al. Hepatitis C virus core protein upregulates the expression of vascular endothelial growth factor via the nuclear factor-B/hypoxia-inducible factor-1alpha axis under hypoxic conditions. Hepatol Res 2012; 42: 591-600.

13. Wilson GK, Brimacombe CL, Rowe IA, et al. A dual role for hypoxia inducible factor-1 alpha in the hepatitis $C$ virus lifecycle and hepatoma migration. J Hepatol 2012; 56: 803-9.

14. Trocha M, Merwid-Ląd A, Pieśniewska M, et al. Age-related differences in function and structure of rat livers subjected to ischemia/reperfusion. Arch Med Sci 2018; 14: 388-95.
15. Gerresheim GK, Bathke J, Michel AM, et al. Cellular gene expression during hepatitis $C$ virus replication as revealed by ribosome profiling. Int J Mol Sci 2019; 20: E1321.

16. Abdel Aziz IM, Badr El-Din NM, Mabrouk MM, et al. Study the serum level of vascular endothelial growth factor receptor 1 (VEGFR1) among patients with hepatocellular carcinoma. Al-Azhar Assiut Med J 2013; 10 (4 Suppl 1): 139-62.

17. Tawfeek NA, EL-Gendy NA, Ibrahim MY, et al. Soluble vascular endothelial growth factor and its soluble receptor 1 in patients with post-hepatitis C virus liver cirrhosis. Med J Cairo Univ 2013; 81: 77-82.

18. Demerdash HM, Hussien HM, Hassouna E, et al. Detection of microRNA in hepatic cirrhosis and hepatocellular carcinoma in hepatitis C genotype-4 in Egyptian patients. Biomed Res Int 2017; 2017: 1806069.

19. Salum GM, Bader El Din NG, et al. Vascular endothelial growth factor expression in hepatitis C virus-induced liver fibrosis: a potential biomarker. J Interferon Cytokine Res 2017; 37: 310-6.

20. Çadırcı K, Havva Keskin H, Okçu N. Vascular endothelial growth factor levels in chronic hepatitis and liver cirrhosis associated with hepatitis C. Kafkas J Med Sci 2019; 9: 74-8.

21. Roth KJ, Copple BL. Role of hypoxia-inducible factors in the development of liver fibrosis. Cell Mol Gastroenterol Hepatol 2015; 1: 589-97.

22. Harma M, Erel O. Measurement of the total antioxidant response in preeclampsia with a novel automated method. Euro J Obst Gyn Reprod Biol 2005; 118: 47-51.

23. Erel O. A novel automated method to measure total antioxidant response against potent free radical reactions. Clin Biochem 2004; 37: 112-9.

24. Jentzsch AM, Bachmann H, Fürst P, Biesalski HK. Improved analysis of malondialdehyde in human body fluids. Free Radic Biol Med 1996; 20: 251-6.

25. Ringelhan M, McKeating JA, Protzer U. Viral hepatitis and liver cancer. Philos Trans R Soc Lond B Biol Sci 2017; 372:pii: 20160274.

26. Anticoli S, Amatore D, Matarrese P, et al. Counteraction of HCV-induced oxidative stress concurs to establish chronic infection in liver cell cultures. Oxid Med Cell Longev 2019; 2019: 6452390.

27. Lozano-Sepulveda SA, Bryan-Marrugo OL, Cordova-Fletes $C$, et al. Oxidative stress modulation in hepatitis $C$ virus infected cells. World J Hepatol 2015; 7: 2880-9.

28. Khedr MA, El-Araby HA, Konsowa HA, et al. Glutathione peroxidase and malondialdehyde in children with chronic hepatitis C. Clin Exp Hepatol 2019; 5: 81-7.

29. Quarato G, Scrima R, Agriesti F, et al. Targeting mitochondria in the infection strategy of the hepatitis $\mathrm{C} \mathrm{vi-}$ rus. Int J Biochem Cell Biol 2013; 45: 156-66.

30. Ripoli M, D’Aprile A, Quarato G, et al. Hepatitis C virus-linked mitochondrial dysfunction promotes hypoxia-inducible factor 1 alpha-mediated glycolytic adaptation. J Virol 2010; 84: 647-60.

31. Zhu C, Liu X, Wang S, et al. Hepatitis C virus core protein induces hypoxia-inducible factor $1 \alpha$-mediated vascular endothelial growth factor expression in Huh7.5.1 cells. Mol Med Rep 2014; 9: 2010-4.

32. Jung GS, Jeon JH, Choi YK, et al. Pyruvate dehydrogenase kinase regulates hepatitis C virus replication. Sci Rep 2016; 6: 30846.

33. Woll PJ, Record CO. Lactate elimination in man: effects of lactate concentration and hepatic dysfunction. Eur J Clin Invest 1979; 9: 397-404. 
34. Welker MW, Luhne S, Lange CM, et al. Lactic acidosis in patients with hepatitis $C$ virus cirrhosis and combined ribavirin/sofosbuvir treatment. J Hepatol 2016; 64: 790-9.

35. Alkharsah KR. VEGF upregulation in viral infections and its possible therapeutic implications. Int J Mol Sci 2018; 19: 1642 .

36. Paternostro C, David E, Novo E, Parola M. Hypoxia, angiogenesis and liver fibrogenesis in the progression of chronic liver diseases. World J Gastroenterol 2010; 16: 281-8.

37. Nasimuzzaman M, Waris G, Mikolon D, Stupack DG, Siddiqui $A$. Hepatitis $C$ virus stabilizes hypoxia-inducible factor 1 and stimulates the synthesis of vascular endothelial growth factor. J Virol 2007; 81: 10249-57.

38. Jaroszewicz J, Januszkiewicz M, Flisiak R, Rogalska $M$, Kalinowska A, Wierzbicka I. Circulating vascular endothelial growth factor and its soluble receptors in patients with liver cirrhosis: possible association with hepatic function impairment. Cytokine 2008; 44: 14-7.

39. Coulon S, Heindryckx F, Geerts A, Van Steenkiste C Colle I, Van Vlierberghe $\mathrm{H}$. Angiogenesis in chronic liver disease and its complications. Liver Int 2010; 31 . 146-62.

40. Sharma BK, Srinivasan R, Kapil S, et al. Angiogenic and anti-angiogenic factor gene transcript level quantitation by quantitative real time PCR in patients with hepatocellular carcinoma. Mol Biol Rep 2013; 40: 5843-52.

41. Elpek GO. Angiogenesis and liver fibrosis. World J Hepatol 2015; 7: 377-91.

42. Helaly GF, Abou Shamaa LA. Influence of hepatitis C virus infection on circulating levels of SICAM-1 and VEGF in patients with hepatitis $C$ and hepatocellular carcinoma (HCC) and their role in enhancing detection of HCC. Egypt J Immunol 2006; 13: 27-38.

43. Reyad S, Hussein HA. Vascular endothelial growth facto as a novel serum marker for hepatocellular carcinoma in Egyptian patients with HCV-related liver cirrhosis. Egypt Liver J 2014; 4: 117-21.

44. Mukozu T, Nagai H, Matsui D, Kanekawa T, Sumino $Y$. Serum VEGF as a tumor marker in patients with HCV-related liver cirrhosis and hepatocellular carcinoma. Anticancer Res 2013; 33: 1013-21.

45. Ratnasari N, Nurdjanah S, Sadewa A, et al. The role of vascular endothelial growth factor $-634 \mathrm{G} / \mathrm{C}$ and its soluble receptor on chronic liver disease and hepatocellular carcinoma. Arab J Gastroenterol 2016; 17: 61-6.

46. Li H, Huang $M H$, Jiang JD, et al. Hepatitis C: from in flammatory pathogenesis to anti-inflammatory/hepatoprotective therapy. World J Gastroenterol 2018; 24 5297-311.

47. Jabłońska J, Pawłowski T, Laskus T, et al. The correlation between pretreatment cytokine expression patterns in peripheral blood mononuclear cells with chronic hepatitis C outcome. BMC Infect Dis 2015; 15: 556

48. Wicks IP, Roberts AW. Targeting GM-CSF in inflammatory diseases. Nat Rev Rheumatol 2016; 12: 37-48.

49. Brem H, Howell R, Criscitelli T, et al. Practical application of granulocyte-macrophage colony-stimulating factor (GM-CSF) in patients with wounds. Surg Technol Int 2018; 32: 61-6.

50. Becher B, Tugues S, Greter M. GM-CSF: from growth factor to central mediator of tissue inflammation. Immunity 2016 ; 45 : 963-73

51. Zahran N, Sayed A, William I, et al. Neutrophil apoptosis: impact of granulocyte macrophage colony stimulating factor on cell survival and viability in chronic kidney disease and hemodialysis patients. Arch Med Sci 2013; 9 984-9.
52. Bahgat MH, Sayed-Ahmed NA, El-Shinnawy H, Nour ElDin MS, Zalata KR. Role of granulocyte macrophage-colony stimulating factor GM-CSF in chronic hepatitis B and $C$ infections. J Med Sci 2001; 1: 1-7.

53. Singh P, González-Ramos S, Mojena M, et al. GM-CSF enhances macrophage glycolytic activity in vitro and improves detection of inflammation in vivo. J Nucl Med 2016; 57: 1428-35.

54. Patel K, Remlinger KS, Walker TG, et al. Multiplex protein analysis to determine fibrosis stage and progression in patients with chronic hepatitis C. Clin Gastroenterol Hepatol 2014; 12: 2113-20.e1-3.

55. Sousa GM, Oliveira IS, Andrade LJ, et al. Serum levels of Th17 associated cytokines in chronic hepatitis $C$ virus infection. Cytokine 2012; 60: 138-42.

56. Bhattacharya P, Budnick I, Singh $M$, et al. Dual role of GM-CSF as a pro-inflammatory and a regulatory cytokine: implications for immune therapy. J Interferon Cytokine Res 2015; 35: 585-99.

57. Masalova OV, Lesnova El, Shingarova LN, et al. The combined application of nucleotide and amino acid sequences of NS3 hepatitis C virus protein, DNA encoding granulocyte macrophage colony-stimulating factor and inhibitor of regulatory $T$ cells induces effective immune response against hepatitis C virus. Mol Biol 2012; 46: 525-34.

58. Fallatah HI, Akbar HO. Elevated serum immunoglobulin $G$ levels in patients with chronic liver disease in comparison to patients with autoimmune hepatitis. Libyan J Med 2010; 5. doi: 10.3402/ljm.v5i0.4857.

59. Maruyama S, Hirayama C, Horie Y, Yorozu K, Maeda K, Inoue $M$. Serum immunoglobulins in patients with chronic hepatitis C: a surrogate marker of disease severity and treatment outcome. Hepatogastroenterology 2007; 54: 493-8.

60. Watt K, Uhanova J, Gong Y, Kaita K, Doucette K, Pettigrew N. Serum immunoglobulins predict the extent of hepatic fibrosis in patients with chronic hepatitis $\mathrm{C}$ virus infection. J Viral Hepat 2004; 11: 251-6.

61. Tanaka S, Okamoto Y, Yamazaki M, Mitani N, Nakqjima Y, Fukui H. Significance of hyperglobulinemia in severe chronic liver diseases with special reference to the correlation between serum globulin/lgG level and ICG clearance. Hepatogastroenterology 2007; 54: 2301-5. 